\title{
Genome-Based Analysis of Klebsiella spp. Isolates from Animals and Food Products in Germany, 2013-2017
}

\author{
Kathleen Klaper ${ }^{1, *}$, Jens Andre Hammerl ${ }^{2}{ }^{\oplus}$, Jörg Rau ${ }^{3} \oplus$, Yvonne Pfeifer ${ }^{1}$ and Guido Werner ${ }^{1}$ \\ 1 Division Nosocomial Pathogens and Antibiotic Resistances, Department of Infectious Diseases, \\ Robert Koch Institute, Wernigerode Branch, 38855 Wernigerode, Germany; pfeifery@rki.de (Y.P.); \\ wernerg@rki.de (G.W.) \\ 2 Unit Epidemiology, Zoonoses and Antimicrobial Resistance, Department Biological Safety, \\ German Federal Institute for Risk Assessment (Bundesinstitut für Risikobewertung [BfR]), \\ 12277 Berlin, Germany; Jens-Andre.Hammerl@bfr.bund.de \\ 3 Chemical and Veterinary Analysis Agency (CVUAS) Stuttgart, 70736 Fellbach, Germany; \\ joerg.rau@cvuas.bwl.de \\ * Correspondence: klaperk@rki.de
}

check for updates

Citation: Klaper, K.; Hammerl, J.A.; Rau, J.; Pfeifer, Y.; Werner, G.

Genome-Based Analysis of Klebsiella spp. Isolates from Animals and Food Products in Germany, 2013-2017. Pathogens 2021, 10, 573. https:// doi.org/10.3390/pathogens10050573

Academic Editor: Thomas Guillard

Received: 14 April 2021

Accepted: 4 May 2021

Published: 8 May 2021

Publisher's Note: MDPI stays neutral with regard to jurisdictional claims in published maps and institutional affiliations.

Copyright: (c) 2021 by the authors. Licensee MDPI, Basel, Switzerland. This article is an open access article distributed under the terms and conditions of the Creative Commons Attribution (CC BY) license (https:// creativecommons.org/licenses/by/ $4.0 /)$

\begin{abstract}
The increase in infections with multidrug-resistant and virulent Klebsiella pneumoniae (K. pneumoniae) strains poses a serious threat to public health. However, environmental reservoirs and routes of transmission for Klebsiella spp. that cause infections in humans and in livestock animals are not well understood. In this study, we aimed to analyze the distribution of antibiotic resistance genes and important virulence determinants ( $y b t$, clb, iro, iuc, rmpA/A2) among 94 Klebsiella spp. isolates from different animal and food sources isolated between 2013 and 2017 in Germany. Antibiotic susceptibility testing was performed, and the genomes were sequenced by Illumina and Nanopore technology. Genetic relationships were assessed by conducting core genome multilocus sequence typing (cgMLST). Kleborate was used to predict resistance and virulence genes; Kaptive was used to derive the capsule types. The results revealed that 72 isolates $(76.6 \%)$ belonged to the K. pneumoniae sensu lato complex. Within this complex, 44 known sequence types (STs), 18 new STs, and 38 capsule types were identified. Extended-spectrum beta-lactamase (ESBL) genes were detected in 16 isolates $(17.0 \%)$ and colistin resistance in one $(1.1 \%)$ K. pneumoniae isolate. Virulence genes were found in 22 K. pneumoniae isolates. Overall, nine (9.6\%) and $18(19.1 \%)$ isolates possessed the genes $y b t$ and iuc, respectively. Notably, aerobactin (iuc lineage 3) was only detected in K. pneumoniae isolates from domestic pigs and wild boars. This study provides a snapshot of the genetic diversity of Klebsiella spp. in animals and food products in Germany. The siderophore aerobactin was found to be more prevalent in K. pneumoniae strains isolated from pigs than other sources. Further investigations are needed to evaluate if pigs constitute a reservoir for iuc lineage 3.
\end{abstract}

Keywords: Klebsiella pneumoniae; antibiotic resistance; virulence genes; aerobactin; pets; livestock; ESBL

\section{Introduction}

Klebsiella ssp. are Gram-negative, rod shaped, facultative anaerobic bacteria that belong to the Enterobacteriaceae family. They are ubiquitous in soil, surface waters, plants, and intestines of animals and humans [1,2]. The Klebsiella pneumoniae (K. pneumoniae) sensu lato complex is clinically the most problematic and comprises the phylogroups $K$. pneumoniae (Kp1), K. quasipneumoniae subsp. quasipneumoniae (Kp2), K. quasipneumoniae subsp. similipneumoniae (Kp4), K. variicola subsp. variicola (Kp3), K. variicola subsp. tropica (Kp5), K. quasivariicola (Kp6), and K. africana (Kp7) and [3,4]. K. pneumoniae is the most prevalent species isolated from human infections $[4,5]$. Classically, K. pneumoniae is considered to be an opportunistic pathogen, causing infections in immunocompromised patients, including urinary tract infections, pneumonia, and bloodstream infections (BSIs) [6,7]. K. pneumoniae is the second most common cause of BSIs caused by Gram-negative bacteria [8]. Due 
to the acquisition of multiple antibiotic resistance genes, the treatment of $K$. pneumoniae infections has become challenging and multidrug-resistant Klebsiella spp. are considered a public health threat-especially for predisposed persons [9-11]. In the last decades, K. pneumoniae has also been reported as a cause of community-acquired infections, including liver abscesses, endophthalmitis, and meningitis in otherwise healthy individuals $[7,12]$. These infections are often caused by hypervirulent isolates representing specific K. pneumoniae lineages, e.g., sequence types ST23 and ST66 [13,14]. Hypervirulent strains possess a distinct portfolio of virulence factors, mainly several siderophore genes, and express specific capsule types (e.g., K1 or K2) [4,15]. Furthermore, hypervirulent K. pneumoniae often demonstrate a hypermucoviscous phenotype that can be determined by a 'string test' on agar plates [16]. Distinct hypervirulent strains that have also acquired carbapenemase genes can enter hospitals and spread among patients. This has mainly been described for China but can also occasionally be observed in Central Europe, including in Germany [17].

In animals, K. pneumoniae can cause infections including pneumonia, mastitis, and bacteremia [18]. While the molecular and epidemiological characteristics of clinically important, multidrug-resistant K. pneumoniae STs in humans are well studied, little is known of the K. pneumoniae population structure in animals [4]. Livestock colonized by K. pneumoniae is thought to be a potential reservoir for antimicrobial resistance determinants and virulence factors and could facilitate the spread to human isolates [19]. The objective of this study was to investigate the molecular-genetic characteristics of Klebsiella spp. isolates from animal and food sources in Germany via whole-genome sequencing (WGS)based analyses.

\section{Results}

\subsection{Klebsiella spp. Population Analysis}

The study included 94 Klebsiella spp. isolates from animals and food sources of which most isolates ( $\mathrm{n}=58 ; 61.7 \%$ ) were from pigs, pork, cattle, and milk. Furthermore, the collection contains isolates from vegetables, pets, livestock, and wild animals (Figure 1). MALDI-TOF biotyping suggested the presence of $69 \mathrm{~K}$. pneumoniae, $21 \mathrm{~K}$. oxytoca, and 4 $K$. variicola, using the commercial MALDI-biotyper database comprising 8468 entries. Subsequent WGS-based analysis revealed that one proposed K. pneumoniae and ten proposed K. oxytoca isolates were mis-assigned and instead belonged to the species K. grimontii, and nine proposed K. oxytoca isolates to the species K. michiganensis. All K. grimontii genomes contained the beta-lactamase gene bla $a_{\mathrm{OXY}} 6$ which is known to be specific to this Klebsiella species [20,21]. Within the K. pneumoniae sensu lato complex, 67 (71.3\%) isolates were identified as K. pneumoniae, four (4.3\%) as K. variicola subsp. variicola and one (1.1\%) as K. quasipneumoniae subsp. similipneumoniae (Figure 1).

\subsection{Klebsiella spp. Antibiotic Resistance and Resistance Genes}

The majority of the 94 study isolates $(n=73,77.7 \%)$ were resistant to ampicillin. Resistance rates to third-generation cephalosporins, fluoroquinolones, and colistin were $5.3 \%, 3.2 \%$, and $1.1 \%$, respectively. Only one isolate showed resistance to all three classes (Supplementary Table S1). The genome-based prediction of resistance genes revealed the presence of various beta-lactamase genes $\left(b l a_{\mathrm{SHV}}, b l a_{\mathrm{LAP}}, b l a_{\mathrm{LEN}}, b l a_{\mathrm{OKP}-\mathrm{B}}, b l a_{\mathrm{OXY}}, b l a_{\mathrm{TEM}}\right)$ in all but three Klebsiella spp. isolates (Table 1). Although only five isolates exhibited an ESBL phenotype (resistance to third-generation cephalosporins), WGS analyses identified $16 \mathrm{~K}$. pneumoniae isolates that carried ESBL genes bla $a_{\mathrm{SHV}-27}(\mathrm{n}=9), b l a_{\mathrm{SHV}-41}(\mathrm{n}=2), b l a_{\mathrm{CTX}-\mathrm{M}-1}$ $(\mathrm{n}=1), b l a_{\mathrm{CTX}-\mathrm{M}-14}(\mathrm{n}=2)$, and bla ${ }_{\mathrm{CTX}-\mathrm{M}-15}(\mathrm{n}=2)$. However, subsequent sequence analysis of the $b l_{\mathrm{SHV}-27}$ and $b l a_{\mathrm{SHV}-41}$ genes revealed a base-pair substitution (A to C) in all promoter sequences of these genes (Figure 2). The combination of the amino acid substitutions in GyrA (S83I) and ParC (S80I) $(\mathrm{n}=2)$ and the acquired resistance genes $q n r B(\mathrm{n}=1)$ and $q n r S$ $(n=1)$ were detected in three isolates with resistance to the fluoroquinolone ciprofloxacin. Sequence analysis of the single colistin-resistant isolate showed that the chromosomal gene 
$m g r B$ was interrupted by an IS1-family transposase gene. Resistance to carbapenems was not observed in the study isolates.

Table 1. Distribution and prevalence of different beta-lactamase genes, including ESBL genes among the 94 Klebsiella spp. isolates from animals and food.

\begin{tabular}{|c|c|}
\hline \multirow[t]{2}{*}{ Beta-Lactam Resistance } & Isolates \\
\hline & $\mathrm{n}=94$ \\
\hline \multicolumn{2}{|c|}{ Beta-lactamase genes } \\
\hline$b l a_{\mathrm{SHV}-1}$ & $9(9.6 \%)$ \\
\hline$b^{b} a_{\mathrm{SHV}-11}$ & $27(28.7 \%)$ \\
\hline$b l a_{\mathrm{SHV}-33}$ & $1(1.1 \%)$ \\
\hline$b l a_{\mathrm{SHV}-37}$ & $2(2.1 \%)$ \\
\hline$b l a_{\mathrm{SHV}-61}$ & $1(1.1 \%)$ \\
\hline$b l a_{\mathrm{SHV}-108}$ & $2(2.1 \%)$ \\
\hline$b l a_{\mathrm{SHV}-119}$ & $1(1.1 \%)$ \\
\hline$b l a_{\mathrm{SHV}-157}$ & $1(1.1 \%)$ \\
\hline$b l a_{\mathrm{SHV}-168}$ & $1(1.1 \%)$ \\
\hline$b l a_{\mathrm{SHV}-187}$ & $1(1.1 \%)$ \\
\hline$b l a_{\mathrm{SHV}-194}$ & $1(1.1 \%)$ \\
\hline$b l a_{\mathrm{SHV}-199}$ & $2(2.1 \%)$ \\
\hline$b l a_{\mathrm{SHV}-211}$ & $1(1.1 \%)$ \\
\hline$b l a_{\mathrm{SHV}-215}$ & $1(1.1 \%)$ \\
\hline$b_{\text {SHV-234 }}$ & $1(1.1 \%)$ \\
\hline$b l a_{\mathrm{SHV}-244}$ & $1(1.1 \%)$ \\
\hline bla $a_{\mathrm{LAP}-2}$ & $2(1.1 \%)$ \\
\hline$b l a_{\mathrm{LEN}-16}$ & $1(1.1 \%)$ \\
\hline$b l a_{\mathrm{LEN}-19}$ & $1(1.1 \%)$ \\
\hline$b l a_{\mathrm{LEN}-24}$ & $1(1.1 \%)$ \\
\hline$b l a_{\mathrm{LEN}-32}$ & $1(1.1 \%)$ \\
\hline$b l a_{\mathrm{OKP}-\mathrm{B}-6}$ & $1(1.1 \%)$ \\
\hline$b_{\text {OXA-1 }}$ & $2(2.1 \%)$ \\
\hline$b l a_{\mathrm{OXY} 1-1}$ & $3(3.2 \%)$ \\
\hline bla $a_{\mathrm{OXY} 1-4}$ & $3(3.2 \%)$ \\
\hline$b l a_{\mathrm{OXY} 1-5}$ & $2(2.1 \%)$ \\
\hline$b l a_{\mathrm{OXY} 2-1}$ & $2(2.1 \%)$ \\
\hline$b l a_{\mathrm{OXY} 5-1}$ & $1(1.1 \%)$ \\
\hline$b l a_{\mathrm{OXY} 6-2}$ & $4(4.3 \%)$ \\
\hline$b l a_{\mathrm{OXY} 6-3}$ & $1(1.1 \%)$ \\
\hline$b l a_{\mathrm{OXY} 6-4}$ & $6(6.4 \%)$ \\
\hline bla $a_{\mathrm{TEM}-1}$ & $3(3.2 \%)$ \\
\hline \multicolumn{2}{|c|}{ Extended-spectrum beta-lactamase genes } \\
\hline bla $a_{\mathrm{SHV}-27}$ * & $9(9.6 \%)$ \\
\hline bla $a_{\mathrm{SHV}-41}$ * & $2(2.1 \%)$ \\
\hline bla $a_{\mathrm{CTX}-\mathrm{M}-1}$ & $1(1.1 \%)$ \\
\hline$b l a_{\mathrm{CTX}}-\mathrm{M}-14$ & $2(2.1 \%)$ \\
\hline$b^{b l a}$ CTX-M-15 & $2(2.1 \%)$ \\
\hline
\end{tabular}

* These isolates did not exhibit the ESBL phenotype with resistance to third-generation cephalosporins due to a base-pair substitution (A to $\mathrm{C}$ ) in the promoter sequences of these genes (Figure 2). 


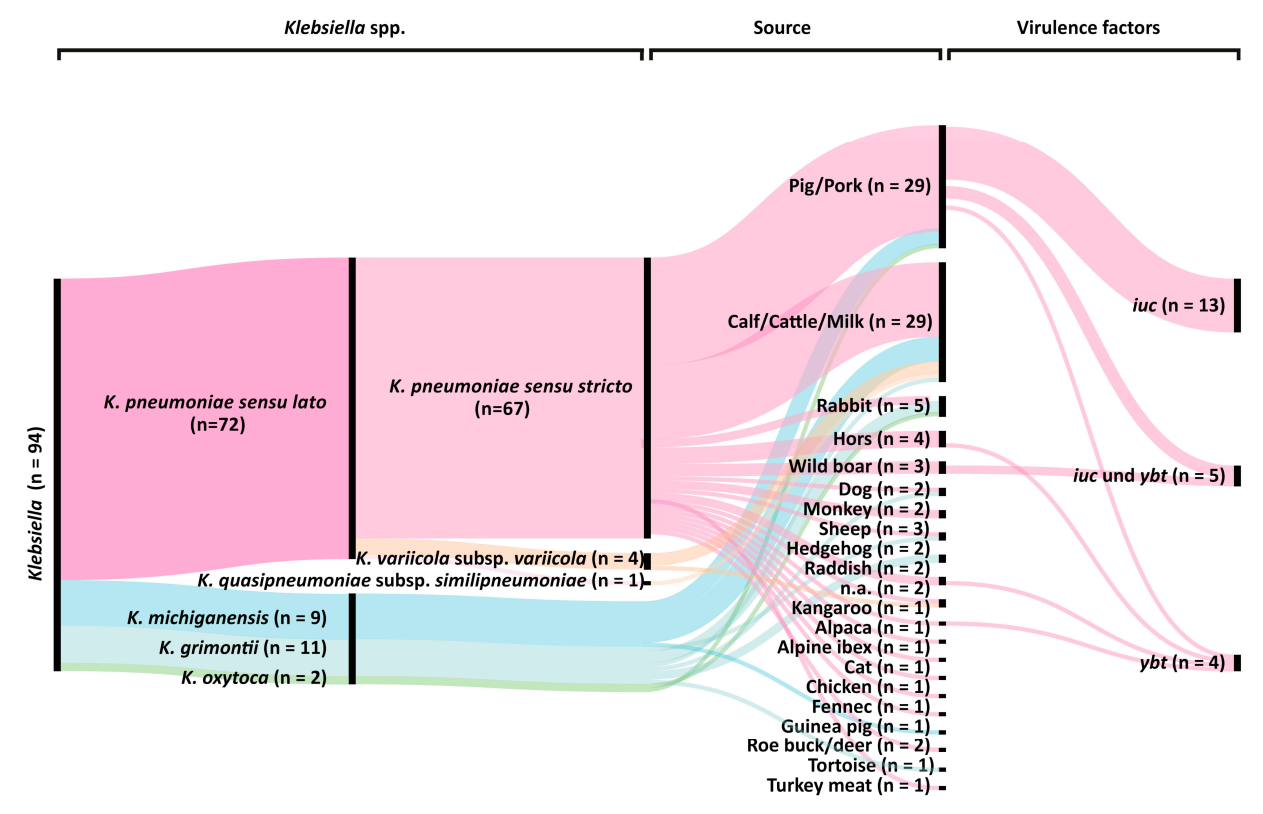

Figure 1. Genome-based classification of the 94 Klebsiella spp. isolates from animals and food. Graph includes species (1st column), phylogroups (2nd column; [3,4]), isolate sources (3rd column) and presence of specific virulence genes (4th column).

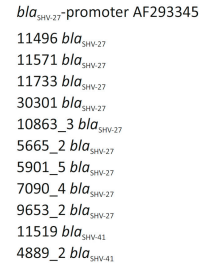

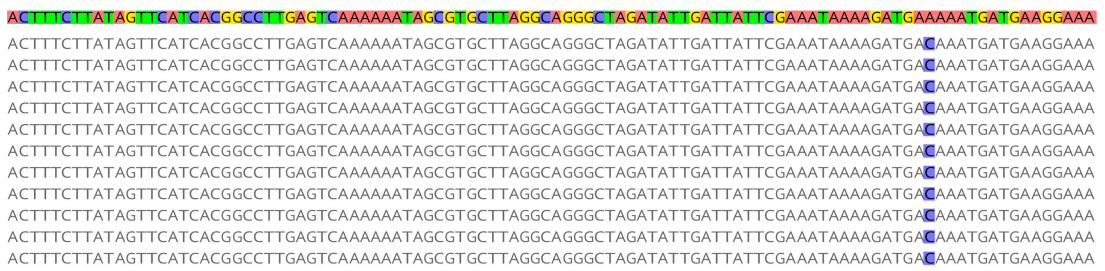

Figure 2. Comparison of the 'wild type' promoter of the ESBL gene bla $a_{\mathrm{SHV}-27}$ (accession no. AF293345) and promoter sequences of $b l a_{\mathrm{SHV}-27 /-41}$ genes in eleven K. pneumoniae isolates of this study. These isolates were susceptible to thirdgeneration cephalosporins (no ESBL phenotype).

\subsection{Molecular Typing}

MLST analysis of the $72 \mathrm{~K}$. pneumoniae sensu lato complex isolates identified $62 \mathrm{STs}$, with ST107 ( $\mathrm{n}=4,5.6 \%$ ) as the most prevalent type (Table 2). Furthermore, 18 new STs were discovered. The genetic diversity was also reflected by capsule locus typing (Supplementary Table S1). The capsule loci KL30 $(\mathrm{n}=7,9.7 \%)$ was the most prevalentout of 38 capsule types (Figure 3). Furthermore, no association of specific ST or capsule type with isolation sources was identified. cgMLST revealed only three small clusters of genetically highly related isolates. Each cluster comprised only two isolates (Figure S1), which did not show more than eight allele differences. Overall, cgMLST analysis revealed no prevalent genetic lineage (Figure 3).

\subsection{Presence of Virulence Factors}

Genetic factors that are associated with hypervirulence were only detected in K. pneumoniae isolates. Overall, $22 \mathrm{~K}$. pneumoniae genomes were positive for one or two siderophore systems (Figure 1). Yersiniabactin (ybt) was present in nine (9.6\%) isolates and 18 isolates (19.1\%) contained aerobactin (iuc) (Supplementary Table S1). Interestingly, aerobactin was only identified in domestic pig and wild boar isolates and belonged to the iuc lineage 3, whereas the presence of yersiniabactin was not associated with a specific isolation source. The addition of long-read sequencing for strain 30312,2 revealed a $171 \mathrm{~kb}$ IncFIB $\mathrm{K}_{\mathrm{K}}$ plasmid carrying $i u c 3$. Subsequent sequence analysis showed that the $\mathrm{IncFIB}_{\mathrm{K}}$ replicon was present in all iuc3 plasmids. Further virulence genes encoding for RmpA/A2, salmochelin, and colibactin were not detected in our study isolates. 
Table 2. Diversity of multilocus sequence types among the $72 \mathrm{~K}$. pneumoniae sensu lato isolates.

\begin{tabular}{|c|c|c|c|}
\hline \multicolumn{2}{|c|}{ Sequence Types } & \multicolumn{2}{|c|}{ Novel Sequence Types } \\
\hline ST & $\mathrm{n}=72$ & ST & $\mathrm{n}=72$ \\
\hline ST11 & $1(1.4 \%)$ & ST5-1LV & $2(2.8 \%)$ \\
\hline ST13 & $1(1.4 \%)$ & ST23-1LV & $1(1.4 \%)$ \\
\hline ST18 & $1(1.4 \%)$ & ST63-1LV & $1(1.4 \%)$ \\
\hline ST20 & $2(2.8 \%)$ & ST70-2LV & $1(1.4 \%)$ \\
\hline ST33 & $1(1.4 \%)$ & ST132-2LV & $1(1.4 \%)$ \\
\hline ST35 & $1(1.4 \%)$ & ST188-1LV & $1(1.4 \%)$ \\
\hline ST39 & $2(2.8 \%)$ & ST200-2LV & $1(1.4 \%)$ \\
\hline ST45 & $2(2.8 \%)$ & ST244-2LV & $1(1.4 \%)$ \\
\hline ST46 & $1(1.4 \%)$ & ST301-3LV & $1(1.4 \%)$ \\
\hline ST107 & $4(5.6 \%)$ & ST464-1LV & $1(1.4 \%)$ \\
\hline ST109 & $1(1.4 \%)$ & ST472-2LV & $1(1.4 \%)$ \\
\hline ST127 & $1(1.4 \%)$ & ST541-1LV & $1(1.4 \%)$ \\
\hline ST147 & $1(1.4 \%)$ & ST919-1LV & $1(1.4 \%)$ \\
\hline ST153 & $1(1.4 \%)$ & ST2397-1LV & $2(2.8 \%)$ \\
\hline ST159 & $1(1.4 \%)$ & ST2404-1LV & $1(1.4 \%)$ \\
\hline ST188 & $1(1.4 \%)$ & ST3377-1LV & $1(1.4 \%)$ \\
\hline ST289 & $1(1.4 \%)$ & ST3381-1LV & $1(1.4 \%)$ \\
\hline ST290 & $2(2.8 \%)$ & ST3526-1LV & $1(1.4 \%)$ \\
\hline ST322 & $1(1.4 \%)$ & & \\
\hline ST323 & $2(2.8 \%)$ & & \\
\hline ST347 & $1(1.4 \%)$ & & \\
\hline ST485 & $1(1.4 \%)$ & & \\
\hline ST494 & $1(1.4 \%)$ & & \\
\hline ST530 & $1(1.4 \%)$ & & \\
\hline ST610 & $1(1.4 \%)$ & & \\
\hline ST611 & $1(1.4 \%)$ & & \\
\hline ST661 & $1(1.4 \%)$ & & \\
\hline ST705 & $1(1.4 \%)$ & & \\
\hline ST991 & $1(1.4 \%)$ & & \\
\hline ST1017 & $1(1.4 \%)$ & & \\
\hline ST1836 & $1(1.4 \%)$ & & \\
\hline ST1838 & $1(1.4 \%)$ & & \\
\hline ST1996 & $1(1.4 \%)$ & & \\
\hline ST2248 & $1(1.4 \%)$ & & \\
\hline ST2426 & $1(1.4 \%)$ & & \\
\hline ST2428 & $1(1.4 \%)$ & & \\
\hline ST2451 & $1(1.4 \%)$ & & \\
\hline ST3073 & $1(1.4 \%)$ & & \\
\hline ST3175 & $1(1.4 \%)$ & & \\
\hline ST3380 & $1(1.4 \%)$ & & \\
\hline ST3839 & $1(1.4 \%)$ & & \\
\hline ST3971 & $1(1.4 \%)$ & & \\
\hline ST4247 & $1(1.4 \%)$ & & \\
\hline ST4248 & $1(1.4 \%)$ & & \\
\hline
\end{tabular}

nLV (locus variant), number of loci this strain differs from the closest reported sequence type (ST) (https: //github.com/katholt/Kleborate/wiki/MLST; v. 0.4.0-beta, accessed on 25 March 2021). 


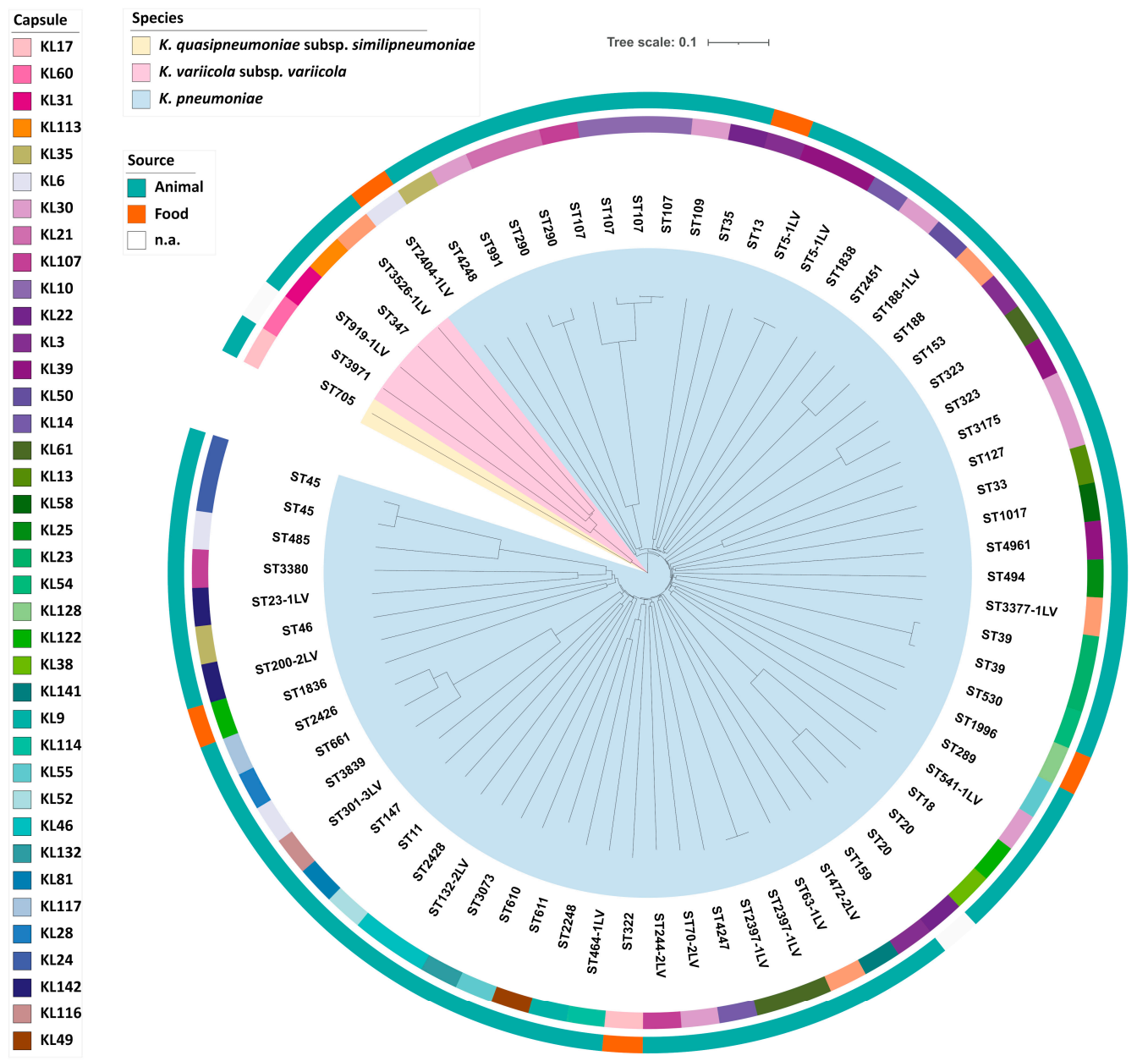

Figure 3. Phylogeny of 72 K. pneumoniae sensu lato isolates. The inner circle shows a Neighbor Joining tree based on cgMLST analysis. Each clade is colored according to the assigned species. Sequence types (STs) are labelled on the tree. The corresponding capsule locus (KL) and isolation sources are color-coded in the two outer rings (see legend).

\section{Discussion}

The analysis of 94 randomly collected Klebsiella spp. isolates from different animals and food sources showed that the species identification was not consistent between the two employed methods. Although MALDI-TOF spectrometry is commonly used in veterinary and medical microbiology laboratories, its discriminatory power depends on the underlying database, which requires continuous curation and updates, especially when closely related taxa such as Klebsiella spp. are analyzed. In this case, WGS-based analyses are better suited to species identification [4,5,22]. In accordance with previous studies, a substantial proportion of the study isolates ( $\mathrm{n}=67,71.3 \%$ ) were classified as K. pneumoniae sensu stricto, suggesting a wide prevalence also in a veterinary context. Such isolates appeared as food colonizers or as infectious agents among livestock animals [23-25].

Klebsiella spp. are rarely detected in animals and food samples [4]. Nevertheless, there are reports of sequence types ST11, ST15, ST25, and ST23 being isolated from livestock and companion animals [26-31]. These globally distributed STs are associated with the majority of nosocomial and community-acquired K. pneumoniae infections in humans [4]. In this study, we detected one K. pneumoniae ST11 and one ST147 isolate in pig and cattle, respectively. Since these STs are proposed to be human-associated, it is conceivable that they were transmitted from humans to animals [32]. Further human-associated or hypervirulent STs were not detected. However, our study collection contains a high genetic diversity, with 62 STs among 67 K. pneumoniae sensu stricto isolates. From this set, no predominant host-associated sequence type or reservoir could be determined. ST107 (four isolates, 
three with capsule type KL10) was the most prevalent ST among the 94 study isolates. K. pneumoniae of this ST have been occasionally isolated from human patients [33-35].

Antibiotic use in veterinary medicine is thought to increase the risk of antibioticresistant bacteria that can be transmitted to humans [36]. High prevalence rates of Escherichia coli with ESBL-mediated resistance to third-generation cephalosporins and MCR-1mediated colistin resistance have been reported from livestock and food in recent years [37-39]. As expected, the resistance to ampicillin was frequent among the study collection due to the presence of intrinsic beta-lactamase genes in Klebsiella spp. (bla $\left.a_{\mathrm{SHV}}, b l a_{\mathrm{OXY}}, b l a_{\mathrm{OKP}}, b l a_{\mathrm{LEN}}\right)$. ESBL genes were detected in 16 of 94 isolates, but only five isolates with $b_{1} a_{\text {CTX-M genes }}$ showed resistance to third-generation cephalosporins (ST11, ST13, ST107, ST1017, ST1836). The remaining eleven isolates harbored $b l a_{\mathrm{SHV}-27}$ and $b l a_{\mathrm{SHV}-41}$ genes, but also a base-pair substitution in their promoter sequence, that has been described to be associated with a weak bla gene expression and non-ESBL phenotype [40]. Resistance to colistin was detected in only one (ST11, pig) of the 94 Klebsiella spp. isolates, but the underlying mechanism was not MCR-1-mediated, as described for E. coli from livestock [39]. Instead, an insertion in the $m g r B$ gene was detected, which results in a truncated and most likely non-functional protein [41]. Changes in the $m g r B$ gene have frequently been described as a cause of colistin resistance in K. pneumoniae [42].

Virulence genes were present in only $22 \mathrm{~K}$. pneumoniae isolates. Interestingly, the iuc gene encoding the siderophore aerobactin was associated with K. pneumoniae isolates of pig and wild boar origin (Figure 1, Table S1). Although these isolates differed in ST and capsule type, all harbored the iuc3 gene. The iuc lineage 3 is associated with self-transmissible $\mathrm{IncFIB}_{\mathrm{K}}$ and $\mathrm{IncFII}_{\mathrm{K}}$ virulence plasmids [43]. Since all $22 \mathrm{iuc3}$ isolates harbored plasmids of the IncFIB $_{K}$ type, we believe that domestic pigs could be a reservoir for K. pneumoniae carrying iuc3 on plasmids. However, this hypothesis requires further experimental proof using larger study collections.

\section{Materials and Methods}

\subsection{Bacterial Isolates}

A total of 90 Klebsiella spp. isolates were collected by the Chemical and Veterinary Analysis Agency Stuttgart between 2013 and 2017 as part of their routine diagnostic activities, one isolate was added from the Bundesinstitut für Risikobewertung (BfR) and three additional isolates from 2004 and 2006, respectively, were included as reference isolates. Further information about the isolates can be found on MALDI-UP [44].

\subsection{Species Identification and Antibiotic Susceptibility Testing (AST)}

Klebsiella species identification was conducted using a Bruker Microflex LT MALDI TOF MS after performing an extraction protocol in combination with the Biotyper database "MBT 8,468 MSP library" as recommended by the manufacturer. The species was assigned if the first two hits matched and showed a score $>2.0$. AST was performed using the broth microdilution assay according to CLSI guidelines. An interpretation of the minimal inhibitory concentrations was carried out using the European Committee on Antimicrobial Susceptibility Testing (EUCLAST) clinical breakpoints v10 derived for the human clinical context (http:/ / www.eucast.org/clinical_breakpoints, accessed on 25 March 2021) (Supplementary Table S1).

\subsection{Whole Genome Sequencing (WGS) and De Novo Assembly}

Bacteria were cultivated at $37^{\circ} \mathrm{C}$ for $16-20 \mathrm{~h}$ under shaking conditions in lysogeny broth (LB). Genomic DNA was extracted from overnight cultures using the Purelink Genomic DNA Mini Kit (Thermo Fisher Scientific Inc., Waltham, MA, USA), in line with the manufacturer's instructions. Quality evaluation and DNA quantification was carried out with the Nanodrop 1000 and the Qubit 4.0 system (Thermo Fisher), respectively. Sequencing libraries were prepared using the Nextera XT DNA Flex Sample Preparation Kit and sequenced using the MiSeq reagent v3 600 cycle kit for paired-end sequence determination 
$(2 \times 300 \mathrm{bp})$ according to the manufacturer's protocol, on a Miseq benchtop device (Illumina Inc., San Diego, CA, USA). Read qualities were assessed using the pipeline QCumber (v2.1.1) where the FastQC (v0.11.5) and Kraken (v0.10.6) tools are implemented [45]. Raw reads were trimmed using Trimmomatic (v0.36; options 'sliding window 4:20') [46] and de novo assembled with SPAdes (v3.12.0) [47]. In addition, an iuc3-positive isolate was subjected to long-read sequencing by MinION (Oxford Nanopore Technologies, Oxford, UK). A hybrid assembly of Illumina and MinION sequence data was done using Unicycler (v0.4.4; https://github.com/rrwick/Unicycler, accessed on 25 March 2021) [48]. The plasmid sequence was annotated by using the Geneious Prime software (v2020.2.3; Biomatters Ltd., Auckland, New Zealand).

\subsection{WGS-Based Typing and Virulence and Resistance Gene Prediction}

For phylogenetic analysis, the Ridom software SeqSphere+ v7.1.0 (Ridom GmbH, Münster, Germany) was used. The assembled genomes were uploaded to for multilocus sequence typing (MLST). New sequence types were assigned through SeqSphere+. The assignment to cluster types was done using a SeqSphere+ defined K. pneumoniae sensu lato core genome MLST (cgMLST) scheme from which a neighbor-joining phylogenetic tree was generated. The resulting tree was visualized with the $i T O L$ software (v5.6.1) [49]. Identification of capsule loci was done using Kaptive (http:/ / kaptive.holtlab. net/, accessed on 25 March 2021). The presence of known virulence factors (clb, ybt, iro, iuc, $r m p A / r m p A 2)$ and various resistance genes was determined with the Kleborate tool (https://github.com/katholt/Kleborate, accessed on 25 March 2021) and SeqSphere+.

\section{Conclusions}

This study demonstrates the genetic diversity of Klebsiella spp. isolates from animals and food products. Despite this diversity, we identified several well-known epidemic clones associated with nosocomial infections and multidrug resistance in humans, such as K. pneumoniae ST11 and ST147. The investigation of antibiotic resistance genes showed that the presence of the ESBL genes bla $a_{\mathrm{SHV}-27}$ and $b a_{\mathrm{SHV}-41}$ did not result in resistance to third generation cephalosporins due to a mutation in their promoter sequences. The virulence factor aerobactin was the most prevalent siderophore locus in our collection, and our data suggest that pig isolates may act as a reservoir for the siderophore iuc lineage 3 in K. pneumoniae.

Supplementary Materials: The following table is available online at https://www.mdpi.com/ article /10.3390 / pathogens10050573/s1, Table S1: Characteristics of 94 K. pneumoniae isolates from different animals and food products, 2013-2017, Germany.

Author Contributions: J.R. provided isolates, source data and performed MALDI TOF MS analysis. J.A.H. performed phenotypic characterization and genome sequencing. K.K. analyzed the WGS data. K.K., J.A.H., Y.P., and G.W. wrote the manuscript and designed the figures and tables. All authors made a substantial, direct, and intellectual contribution to the work, in interpreting results, providing critical feedback, and finalizing the manuscript. All authors have read and agreed to the published version of the manuscript.

Funding: The study received support by a research grant from the Robert Koch Institute as part of the GOHI initiative (https:/ / www.gohi.online/GOHI/EN/Home/Homepage_node.html, accessed on 25 March 2021) to G.W. and K.K. Whole genome sequencing of the Klebsiella isolates was financially supported by the BfR to JAH (grant 1322-648).

Institutional Review Board Statement: Not applicable.

Informed Consent Statement: Not applicable.

Data Availability Statement: Information about the isolates used and the MALDI-TOF MS single spectra are listed on the MALDI-UP website https://maldi-up.ua-bw.de/, accessed on 25 March 2021 [44]. The nucleotide sequences of the Klebsiella spp. isolates of this study are deposited in GenBank. The accession numbers are specified in Supplementary Table S1. 
Acknowledgments: The authors thank Silvia Schmoger (BfR) for excellent technical assistance and WGS, Martin Dyk (CVUAS) for technical assistance with MALDI-TOF MS. We also thank Christopher F. Schuster and Robert Weber (RKI) for MinION sequencing and Christopher F. Schuster for critically reading and English editing of the manuscript.

Conflicts of Interest: The authors declare no conflict of interest.

\section{References}

1. Podschun, R.; Ullmann, U. Klebsiella spp. as nosocomial pathogens: Epidemiology, taxonomy, typing methods, and pathogenicity factors. Clin. Microbiol. Rev. 1998, 11, 589-603. [CrossRef]

2. Davis, G.S.; Waits, K.; Nordstrom, L.; Weaver, B.; Aziz, M.; Gauld, L.; Grande, H.; Bigler, R.; Horwinski, J.; Porter, S.; et al. Intermingled Klebsiella pneumoniae Populations between Retail Meats and Human Urinary Tract Infections. Clin. Infect. Dis. 2015, 61, 892-899. [CrossRef]

3. Rodriguez-Medina, N.; Barrios-Camacho, H.; Duran-Bedolla, J.; Garza-Ramos, U. Klebsiella variicola: An emerging pathogen in humans. Emerg. Microbes Infect. 2019, 8, 973-988. [CrossRef] [PubMed]

4. Wyres, K.L.; Lam, M.M.C.; Holt, K.E. Population genomics of Klebsiella pneumoniae. Nat. Rev. Microbiol. 2020, 18, 344-359. [CrossRef] [PubMed]

5. Rodrigues, C.; Passet, V.; Rakotondrasoa, A.; Brisse, S. Identification of Klebsiella pneumoniae, Klebsiella quasipneumoniae, Klebsiella variicola and Related Phylogroups by MALDI-TOF Mass Spectrometry. Front. Microbiol. 2018, 9, 3000. [CrossRef] [PubMed]

6. Paczosa, M.K.; Mecsas, J. Klebsiella pneumoniae: Going on the Offense with a Strong Defense. Microbiol. Mol. Biol. Rev. 2016, 80, 629-661. [CrossRef] [PubMed]

7. Martin, R.M.; Bachman, M.A. Colonization, Infection, and the Accessory Genome of Klebsiella pneumoniae. Front. Cell. Infect. Microbiol. 2018, 8, 4. [CrossRef] [PubMed]

8. Tian, L.; Tan, R.; Chen, Y.; Sun, J.; Liu, J.; Qu, H.; Wang, X. Epidemiology of Klebsiella pneumoniae bloodstream infections in a teaching hospital: Factors related to the carbapenem resistance and patient mortality. Antimicrob. Resist. Infect. Control 2016, 5, 48. [CrossRef]

9. Tanner, W.D.; VanDerslice, J.A.; Goel, R.K.; Leecaster, M.K.; Fisher, M.A.; Olstadt, J.; Gurley, C.M.; Morris, A.G.; Seely, K.A.; Chapman, L.; et al. Multi-state study of Enterobacteriaceae harboring extended-spectrum beta-lactamase and carbapenemase genes in US drinking water. Sci. Rep. 2019, 9, 3938. [CrossRef]

10. Effah, C.Y.; Sun, T.W.; Liu, S.H.; Wu, Y.J. Klebsiella pneumoniae: An increasing threat to public health. Ann. Clin. Microbiol. Antimicrob. 2020, 19, 1-9. [CrossRef]

11. Dinkelacker, A.G.; Vogt, S.; Oberhettinger, P.; Mauder, N.; Rau, J.; Kostrzewa, M.; Rossen, J.W.A.; Autenrieth, I.B.; Peter, S.; Liese, J. Typing and Species Identification of Clinical Klebsiella Isolates by Fourier Transform Infrared Spectroscopy and Matrix-Assisted Laser Desorption Ionization-Time of Flight Mass Spectrometry. J. Clin. Microbiol. 2018, 56, e00843-18. [CrossRef]

12. Wang, J.H.; Liu, Y.C.; Lee, S.S.J.; Yen, M.Y.; Chen, Y.S.; Wang, J.H.; Wann, S.R.; Lin, H.H. Primary liver abscess due to Klebsiella pneumoniae in Taiwan. Clin. Infect. Dis. 1998, 26, 1434-1438. [CrossRef] [PubMed]

13. Bialek-Davenet, S.; Criscuolo, A.; Ailloud, F.; Passet, V.; Jones, L.; Delannoy-Vieillard, A.S.; Garin, B.; Le Hello, S.; Arlet, G.; Nicolas-Chanoine, M.H.; et al. Genomic definition of hypervirulent and multidrug-resistant Klebsiella pneumoniae clonal groups. Emerg. Infect. Dis. 2014, 20, 1812-1820. [CrossRef]

14. Tang, M.; Kong, X.; Hao, J.; Liu, J. Epidemiological Characteristics and Formation Mechanisms of Multidrug-Resistant Hypervirulent Klebsiella pneumoniae. Front. Microbiol. 2020, 11, 581543. [CrossRef] [PubMed]

15. Russo, T.A.; Marr, C.M. Hypervirulent Klebsiella pneumoniae. Clin. Microbiol. Rev. 2019, 32. [CrossRef]

16. Shon, A.S.; Bajwa, R.P.S.; Russo, T.A. Hypervirulent (hypermucoviscous) Klebsiella pneumoniae A new and dangerous breed. Virulence 2013, 4, 107-118. [CrossRef] [PubMed]

17. Heiden, S.E.; Hubner, N.O.; Bohnert, J.A.; Heidecke, C.D.; Kramer, A.; Balau, V.; Gierer, W.; Schaefer, S.; Eckmanns, T.; Gatermann, S.; et al. A Klebsiella pneumoniae ST307 outbreak clone from Germany demonstrates features of extensive drug resistance, hypermucoviscosity, and enhanced iron acquisition. Genome Med. 2020, 12, 113. [CrossRef] [PubMed]

18. Roberts, D.E.; McClain, H.M.; Hansen, D.S.; Currin, P.; Howerth, E.W. An outbreak of Klebsiella pneumoniae infection in dogs with severe enteritis and septicemia. J. Vet. Diagn. Investig. 2000, 12, 168-173. [CrossRef]

19. Manges, A.R. Genomic Epidemiology: Revealing Hidden Reservoirs for Klebsiella pneumoniae. Clin. Infect. Dis. 2015, 61, 900-902. [CrossRef]

20. Liu, L.; Feng, Y.; Hu, Y.Y.; Kang, M.; Xie, Y.; Zong, Z.Y. Klebsiella grimontii, a New Species Acquired Carbapenem Resistance. Front. Microbiol. 2018, 9, 2170. [CrossRef]

21. Passet, V.; Brisse, S. Description of Klebsiella grimontii sp nov. Int. J. Syst. Evol. Microbiol. 2018, 68, 377-381. [CrossRef]

22. Pinto, N.A.; D'Souza, R.; Hwang, I.S.; Choi, J.; In, Y.H.; Park, H.S.; Ryu, C.M.; Yong, D.; Lee, K. Whole genome and transcriptome analysis reveal MALDI-TOF MS and SDS-PAGE have limited performance for the detection of the key outer membrane protein in carbapenem-resistant Klebsiella pneumoniae isolates. Oncotarget 2017, 8, 84818-84826. [CrossRef] [PubMed]

23. Guo, Y.; Zhou, H.; Qin, L.; Pang, Z.; Qin, T.; Ren, H.; Pan, Z.; Zhou, J. Frequency, Antimicrobial Resistance and Genetic Diversity of Klebsiella pneumoniae in Food Samples. PLoS ONE 2016, 11, e0153561. [CrossRef] 
24. Zhang, S.; Yang, G.; Ye, Q.; Wu, Q.; Zhang, J.; Huang, Y. Phenotypic and Genotypic Characterization of Klebsiella pneumoniae Isolated From Retail Foods in China. Front. Microbiol. 2018, 9, 289. [CrossRef]

25. Davis, G.S.; Price, L.B. Recent Research Examining Links Among Klebsiella pneumoniae from Food, Food Animals, and Human Extraintestinal Infections. Curr. Environ. Health Rep. 2016, 3, 128-135. [CrossRef] [PubMed]

26. Anzai, E.K.; de Souza, J.C.; Peruchi, A.R.; Fonseca, J.M.; Gumpl, E.K.; Pignatari, A.C.C.; Hirano, Z.M.B.; Silveira, A.C.D. First case report of non-human primates (Alouatta clamitans) with the hypervirulent Klebsiella pneumoniae serotype K1 strain ST 23: A possible emerging wildlife pathogen. J. Med. Primatol. 2017, 46, 337-342. [CrossRef]

27. Bowring, B.G.; Fahy, V.A.; Morris, A.; Collins, A.M. An unusual culprit: Klebsiella pneumoniae causing septicaemia outbreaks in neonatal pigs? Vet. Microbiol. 2017, 203, 267-270. [CrossRef]

28. Marques, C.; Betas, A.; Aboim, C.; Cavaco-Silva, P.; Trigueiro, G.; Gama, L.T.; Pomba, C. Evidence of Sharing of Klebsiella pneumoniae Strains between Healthy Companion Animals and Cohabiting Humans. J. Clin. Microbiol. 2019, 57. [CrossRef]

29. Marques, C.; Menezes, J.; Belas, A.; Aboim, C.; Cavaco-Silva, P.; Trigueiro, G.; Gama, L.T.; Pomba, C. Klebsiella pneumoniae causing urinary tract infections in companion animals and humans: Population structure, antimicrobial resistance and virulence genes. J. Antimicrob. Chemother. 2019, 74, 594-602. [CrossRef]

30. Navon-Venezia, S.; Kondratyeva, K.; Carattoli, A. Klebsiella pneumoniae: A major worldwide source and shuttle for antibiotic resistance. FEMS Microbiol. Rev. 2017, 41, 252-275. [CrossRef]

31. Zhang, R.M.; Li, J.Y.; Wang, Y.; Shen, J.Z.; Shen, Z.Q.; Wang, S.L. Presence of NDM in non-E. coli Enterobacteriaceae in the poultry production environment. J. Antimicrob. Chemother. 2019, 74, 2209-2213. [CrossRef]

32. Ovejero, C.M.; Escudero, J.A.; Thomas-Lopez, D.; Hoefer, A.; Moyano, G.; Montero, N.; Martin-Espada, C.; Gonzalez-Zorn, B. Highly Tigecycline-Resistant Klebsiella pneumoniae Sequence Type 11 (ST11) and ST147 Isolates from Companion Animals. Antimicrob. Agents Chemother. 2017, 61, e02640-16. [CrossRef]

33. Wang, X.; Li, H.; Zhao, C.; Chen, H.; Liu, J.; Wang, Z.; Wang, Q.; Zhang, Y.; He, W.; Zhang, F.; et al. Novel NDM-9 metallobeta-lactamase identified from a ST107 Klebsiella pneumoniae strain isolated in China. Int. J. Antimicrob. Agents 2014, 44, 90-91. [CrossRef]

34. Patil, S.; Chen, X.; Wen, F. Exploring the phenotype and genotype of multi-drug resistant Klebsiella pneumoniae harbouring blaCTX-M group extended-spectrum beta-lactamases recovered from paediatric clinical cases in Shenzhen, China. Ann. Clin. Microbiol. Antimicrob. 2019, 18, 32. [CrossRef] [PubMed]

35. Ku, Y.H.; Chuang, Y.C.; Chen, C.C.; Lee, M.F.; Yang, Y.C.; Tang, H.J.; Yu, W.L. Klebsiella pneumoniae Isolates from Meningitis: Epidemiology, Virulence and Antibiotic Resistance. Sci. Rep. 2017, 7, 6634. [CrossRef]

36. Aarestrup, F.M.; McDermott, P.F.; Wegener, H.C. Transmission of Antibiotic Resistance from Food Animals to Humans. In Campylobacter, 3rd ed.; ASM Press: Washington, DC, USA, 2008; pp. 645-665.

37. Rahman, M.M.; Husna, A.; Elshabrawy, H.A.; Alam, J.; Runa, N.Y.; Badruzzaman, A.T.M.; Banu, N.A.; Al Mamun, M.; Paul, B.; Das, S.; et al. Isolation and molecular characterization of multidrug-resistant Escherichia coli from chicken meat. Sci. Rep. 2020, 10, 21999. [CrossRef]

38. Vidovic, N.; Vidovic, S. Antimicrobial Resistance and Food Animals: Influence of Livestock Environment on the Emergence and Dissemination of Antimicrobial Resistance. Antibiotics 2020, 9, 52. [CrossRef] [PubMed]

39. Irrgang, A.; Roschanski, N.; Tenhagen, B.A.; Grobbel, M.; Skladnikiewicz-Ziemer, T.; Thomas, K.; Roesler, U.; Kasbohrer, A. Prevalence of mcr-1 in E. coli from Livestock and Food in Germany, 2010-2015. PLoS ONE 2016, 11, e0159863. [CrossRef] [PubMed]

40. Zhai, Y.; Zhang, Z.; Wang, Z.; Chen, Y.; Wang, Q.; Lv, Y.; Yang, J.; Zhao, T.; Guo, Y.; Gao, Z. Relative Strengths and Regulation of Different Promoter-Associated Sequences for Various bla $a_{\mathrm{SHV}}$ Genes and Their Relationships to $\beta$-Lactam Resistance. J. Mol. Microbiol. Biotechnol. 2017, 27, 91-101. [CrossRef] [PubMed]

41. Haeili, M.; Javani, A.; Moradi, J.; Jafari, Z.; Feizabadi, M.M.; Babaei, E. MgrB Alterations Mediate Colistin Resistance in Klebsiella pneumoniae Isolates from Iran. Front. Microbiol. 2017, 8, 2470. [CrossRef]

42. Prashar, A.; Bhatia, S.; Gigliozzi, D.; Martin, T.; Duncan, C.; Guyard, C.; Terebiznik, M.R. Filamentous morphology of bacteria delays the timing of phagosome morphogenesis in macrophages. J. Cell Biol. 2013, 203, 1081-1097. [CrossRef]

43. Lam, M.M.C.; Wyres, K.L.; Judd, L.M.; Wick, R.R.; Jenney, A.; Brisse, S.; Holt, K.E. Tracking key virulence loci encoding aerobactin and salmochelin siderophore synthesis in Klebsiella pneumoniae. Genome Med. 2018, 10. [CrossRef] [PubMed]

44. Rau, J.; Eisenberg, T.; Männig, A.; Wind, C.; Lasch, P.; Sting, R. MALDI-UP-An internet platform for the exchange of MALDI-TOF mass spectra. Asp. Food Control Anim. Health (ejournal) 2016, 2016, 1-17.

45. Neumann, B.; Rackwitz, W.; Hunfeld, K.P.; Fuchs, S.; Werner, G.; Pfeifer, Y. Genome sequences of two clinical Escherichia coli isolates harboring the novel colistin-resistance gene variants mcr-1.26 and mcr-1.27. Gut Pathog. 2020, 12, 40. [CrossRef] [PubMed]

46. Bolger, A.M.; Lohse, M.; Usadel, B. Trimmomatic: A flexible trimmer for Illumina sequence data. Bioinformatics 2014, 30, 2114-2120. [CrossRef]

47. Bankevich, A.; Nurk, S.; Antipov, D.; Gurevich, A.A.; Dvorkin, M.; Kulikov, A.S.; Lesin, V.M.; Nikolenko, S.I.; Pham, S.; Prjibelski, A.D.; et al. SPAdes: A new genome assembly algorithm and its applications to single-cell sequencing. J. Comput. Biol. 2012, 19, 455-477. [CrossRef] [PubMed] 
48. Wick, R.R.; Judd, L.M.; Gorrie, C.L.; Holt, K.E. Unicycler: Resolving bacterial genome assemblies from short and long sequencing reads. PLoS Comput. Biol. 2017, 13, e1005595. [CrossRef]

49. Letunic, I.; Bork, P. Interactive Tree Of Life (iTOL) v4: Recent updates and new developments. Nucleic Acids Res. 2019, 47, W256-W259. [CrossRef] [PubMed] 